\title{
Latchbox performance as affected by medial thalamic and habenular lesions in rats
}

\author{
ROBERT THOMPSON \\ Fairview State Hospital, Costa Mesa, California 92626 \\ and University of California Irvine Medical Center, Orange, California 92668
}

and

\author{
RODNEY B. AMRHEIN and CAROL A. HILLIARD \\ Louisiana State University, Baton Rouge, Louisiana 70803
}

\begin{abstract}
Adult albino rats, previously trained on two latchbox tasks, sustained discrete bilateral lesions to either the habenular complex or the medial thalamus and subsequently were tested for retention. No significant deficits on the most difficult task were associated with damage to either the habenular nuclei or the mediodorsal thalamic nucleus. On the other hand, significant deficits were observed in the presence of ventromedial thalamic damage, the magnitude of the retention loss being a function of the extent of damage to the ventromedial thalamic nucleus.
\end{abstract}

It has recently been reported that rats prepared with medial thalamic lesions show significant disturbances in the performance of preoperatively acquired latchbox tasks (Thompson, Gates, \& Gross, 1979). Because the lesions investigated in this study tended to be large, it was not possible to determine with any degree of confidence whether dorsomedial thalamic damage produced greater, lesser, or equivalent deficits in latchbox performance relative to ventromedial thalamic damage. One purpose of the current study was to determine if a critical medial thalamic focus for latchbox deficits exists in the rat and, if it does, whether this focus occupies the dorsomedial, ventromedial, or intermediate sector of the thalamus.

Another objective of the present experiment was to assess the effects of habenular lesions on retention of latchbox tasks in the rat. Selection of this epithalamic complex for study was based upon two recent observations. First, both latchbox (Thompson et al., 1979) and card-displacement (Thompson, 1979) deficits have been noted in rats suffering medial thalamic lesions which extensively invaded the overlying habenular nuclei. Second, the habenular complex is the recipient of a contingent of fibers from the entopeduncular nucleus (Carter \& Fibiger, 1978; Herkenham \& Nauta, 1977). This observation is significant because lesions in the region of the entopeduncular nucleus (the internal segment of the globus pallidus in nonprimate mammals) interfere with latchbox (Thompson et al., 1979) and carddisplacement (Thompson, 1978) performance in rats.

\section{METHOD}

Subjects

Adult male Wistar albino rats (90-110 days of age) were used. All animals were subjected to 3-5 days of handling and a 23-5/6-h water-deprivation schedule prior to preliminary training. The water-deprivation schedule was continued until the completion of original learning and reintroduced 3-5 days before the onset of postoperative testing.

\section{Apparatus}

The apparatus consisted of a startbox and a goalbox, each with a one-way-vision window for monitoring the animal's movements. Interchangeable Plexiglas frames, each containing a Plexiglas door, could be inserted between the startbox and goalbox. A frame containing a door with no latch was used during preliminary training, while frames containing doors with latches were used during formal experimental training. Problem A consisted of a button latch which had to be rotated from the horizontal to the vertical position (in a clockwise direction only) in order to unlock the door. Problem B consisted of a barrel-bolt latch which had to be slid to the right at least $1.7 \mathrm{~cm}$ in order to unlock the door. Photographs of the apparatus and latches may be seen elsewhere (Spiliotis \& Thompson, 1973).

\section{Procedure}

\section{Preliminary Training}

On the 1st day, each rat was allowed to explore the apparatus for $10 \mathrm{~min}$. During this time, the door between the startbox and the goalbox was fully opened and water was available from an aluminum tube located at the far end of the goalbox. This procedure was repeated on the 2 nd day. (Throughout the preliminary training, original learning and relearning periods, the only water available to the animal was located in the apparatus.)

On the 3rd day, the animal was required to push open the door in order to gain access to the goalbox. Upon entering the 
goalbox, the animal was allowed to drink water for $10 \mathrm{sec}$. The animal was then transferred to a restraining cage to await the next trial. Ten trials were usually given with an intertrial interval of 30-120 sec. On the 10th trial, the animal was allowed to drink to satiation provided that its motivational level was high during the preceding nine trials. (In most cases, the rat readily opened the door on all trials and required no further training on this phase of the problem.)

\section{Acquisition}

Each animal was required to learn Problem A and then Problem B. In most cases, 10-15 trials were given daily, with an intertrial interval of 30-120 sec. A trial was initiated when the animal was placed into the startbox and was terminated when the animal either successfully operated the latch, opened the door, and drank for $10 \mathrm{sec}$ or failed to operate the latch within $180 \mathrm{sec}$. At the completion of the trial, the animal was removed from the goalbox (or startbox) and returned to the restraining cage to await the next trial.

A correct response was defined as the unlocking of the latch and the opening of the door within $5 \mathrm{sec}$ after initial contact with any part of the Plexiglas frame, door, or latch. The criterion of learning consisted of at least 9 correct responses within a series of 10 trials in one daily session. A more detailed description of the training procedure has been presented earlier (Thompson et al., 1979).

Upon learning Problem B, each animal was placed on ad-lib food and water for 3-5 days, after which it underwent surgery.

\section{Retention Test}

Following a 2-3-week recovery period, each animal was required to relearn the two problems in the order in which they were originally mastered. Postoperative testing involved the same procedure as that described in original learning.

Retention of each problem was measured in terms of percentage error savings scores using the conventional formula.

\section{Surgery}

The majority of animals sustained bilateral one-stage diencephalic lesions under deep chloral hydrate anesthesia. Details of the surgical procedure may be found elsewhere (Thompson, 1978). Eight animals served as the operated control group, undergoing anesthetization, shaving of hair over the cranium, placement into the headholder, exposure of the skull, and suturing without any further treatment.

\section{Thalamic Groape}

The medial thalamus was arbitrarily divided into three sectors (dorsal, intermediate, and ventral), and different groups of rats were designated to receive lesions to a given sector. The dorsal thalamic group (Group DT) sustained lesions dorsal to the massa intermedia, the ventral thalamic group (Group VT) received lesions ventral to the massa intermedia, and the intermediate thalamic group (Group IT) was subjected to lesions of the massa intermedia which invaded thalamic tissue both dorsal and ventral to this commissure.

A constant anodal current (2.4 mA for Groups DT and IT and $2.0 \mathrm{~mA}$ for Group VT) for a duration of $10 \mathrm{sec}$ was passed through a stainless steel electrode with $1.0 \mathrm{~mm}$ of the tip exposed. Based upon a modified version of the Massopust rat atlas (Thompson, 1978), the frontal (4.0 mm rostral to lambda) and lateral (1.0 mm lateral to the longitudinal sinus) coordinates were the same for all groups; only the ventral coordinate differed (ranging from 6.0 to $7.5 \mathrm{~mm}$ beneath the cortical surface).

\section{Habenular Group}

A separate group of animals was designated to receive lesions to the habenular complex (Group H). In this case, a constant anodal current of $2.4 \mathrm{~mA}$ for a duration of $10 \mathrm{sec}$ was passed through the electrode with $1.0 \mathrm{~mm}$ of the tip exposed. The coordinates were as follows: $+4.0 \mathrm{~mm}$ anterior, $.75 \mathrm{~mm}$ lateral, and $5.0 \mathrm{~mm}$ ventral.

\section{Histology}

At the conclusion of postoperative testing, each brain-damaged rat was killed with an overdose of chloral hydrate. Its vascular system was then perfused with normal saline followed by $10 \%$ Formalin, and the brain was removed and stored in 10\% Formalin for 2-4 days. Each brain was subsequently blocked, frozen, and sectioned frontally at $90 \mu$. Every fourth section through the lesion was preserved and photographed at $12 \times$ with a Leitz enlarger.

From these photographs, the lesions suffered by each animal composing the thalamic groups were reconstructed on work sheets containing three frontal sections through the thalamus (Figures 2, 3 , and 4). This was done for the purpose of estimating the amount of bilateral damage to the thalamus.From these estimates, rankorder correlations were computed between the overall size of the lesions and the extent of the retention losses on Problems $\mathbf{A}$ and B. In order to delineate a medial thalamic focus for latchbox deficits, the photograph of the brain section through the "center" of the lesion was selected for each rat. The central necrotic zone of the lesion (the vacuolated and severely coagulated areas) was then reconstructed on a work sheet (derived from $F=4.0 \mathrm{~mm}$ in the Thompson, 1978, atlas) outlining the boundaries of the mediodorsal, intralaminar (central medial, paracentral, and central lateral), and ventromedial thalamic nuclei (Figure 5). Estimates of the amount of damage to each nucleus were obtained by using a transparent grid divided into $2.0 \mathrm{~mm}$ squares. Separate rankorder correlations were then computed between the amount of damage to a given nucleus and the extent of the retention losses on Problems A and B.

\section{RESULTS}

\section{Original Learning}

All animals, except five, succeeded in reaching the criterion of learning within 70 trials on each of the two latchbox problems. Of the five animals failing to achieve the criterion, three were terminated on Problem A (due largely to timidity) and two were terminated on Problem B. The data derived from these five subjects were not included in any of the analyses to follow.

Table 1 shows the mean errors to reach the criterion of learning on Problems A and B. The Wilcoxon test for paired replicates disclosed that Problem B was significantly more difficult to master than Problem A $(p<.01)$. This finding agrees with earlier reports (Spiliotis \& Thompson, 1973; Thompson et al., 1979).

Table 1

Mean Errors to Criterion and the Percentage of Subjects Using a Particular Response Strategy

\begin{tabular}{cccccc}
\hline & & \multicolumn{4}{c}{ Response Strategy } \\
\cline { 3 - 6 } Problem & Errors & Nose & Teeth & Forepaw & C* $^{*}$ \\
\hline A & 4.7 & 45 & 22 & 15 & 18 \\
B & 9.0 & 11 & 60 & 18 & 11 \\
\hline
\end{tabular}

*Combination. 
Table 1 also shows that most of the subjects used their noses to operate the button latch (Problem A), but preferred to use their teeth to operate the bolt latch (Problem B). These observations also agree with the earlier studies mentioned above.

\section{Postoperative Retention}

\section{Control Group}

Seven of the eight control animals earned savings scores in excess of $65 \%$ on Problem A, but all achieved savings scores of $90 \%$ or better on Problem B. As noted in Table 2, most of the control and (braindamaged) rats used the same manipulative act to operate each latch during the criterion run in the retention test that was used during the criterion run in original learning.

\section{Habenular Group}

Since a few animals with thalamic lesions suffered additional damage to the habenular complex, it would seem appropriate to present the results derived from Group $\mathrm{H}$ first.
Table 2

Mean Retention Scores (Percentage Error Savings) and Percentage of Subjects Changing Their Response Strategy for All Groups

\begin{tabular}{lcrrrrr}
\hline & & \multicolumn{2}{c}{ Problem A } & & \multicolumn{2}{c}{ Problem B } \\
\cline { 6 - 7 } Group & $\mathrm{N}$ & Savings & Change & & Savings & Change \\
\hline Control & 8 & 79.9 & 12.5 & & 97.1 & .0 \\
H & 4 & 66.8 & .0 & 97.2 & .0 \\
DT & 6 & $-30.5^{*}$ & 33.3 & 86.3 & 16.7 \\
IT & 6 & $-261.7^{*}$ & 16.7 & $-60.0^{*}$ & 16.7 \\
VT & 4 & $-45.0^{*}$ & .0 & $-127.0^{*}$ & 25.0 \\
\hline
\end{tabular}

*Differed from the controls at least at the .05 level (MannWhitney U test).

Four rats suffered extensive damage to the lateral and medial habenular nuclei (Figure 1). One earned a savings score of $0 \%$ on Problem A, but subsequently showed perfect retention of Problem B. The remaining three animals earned savings scores in excess of $65 \%$ on each of the two problems. As a group, these animals were not significantly different from the controls in retention of either Problem A or Problem B.

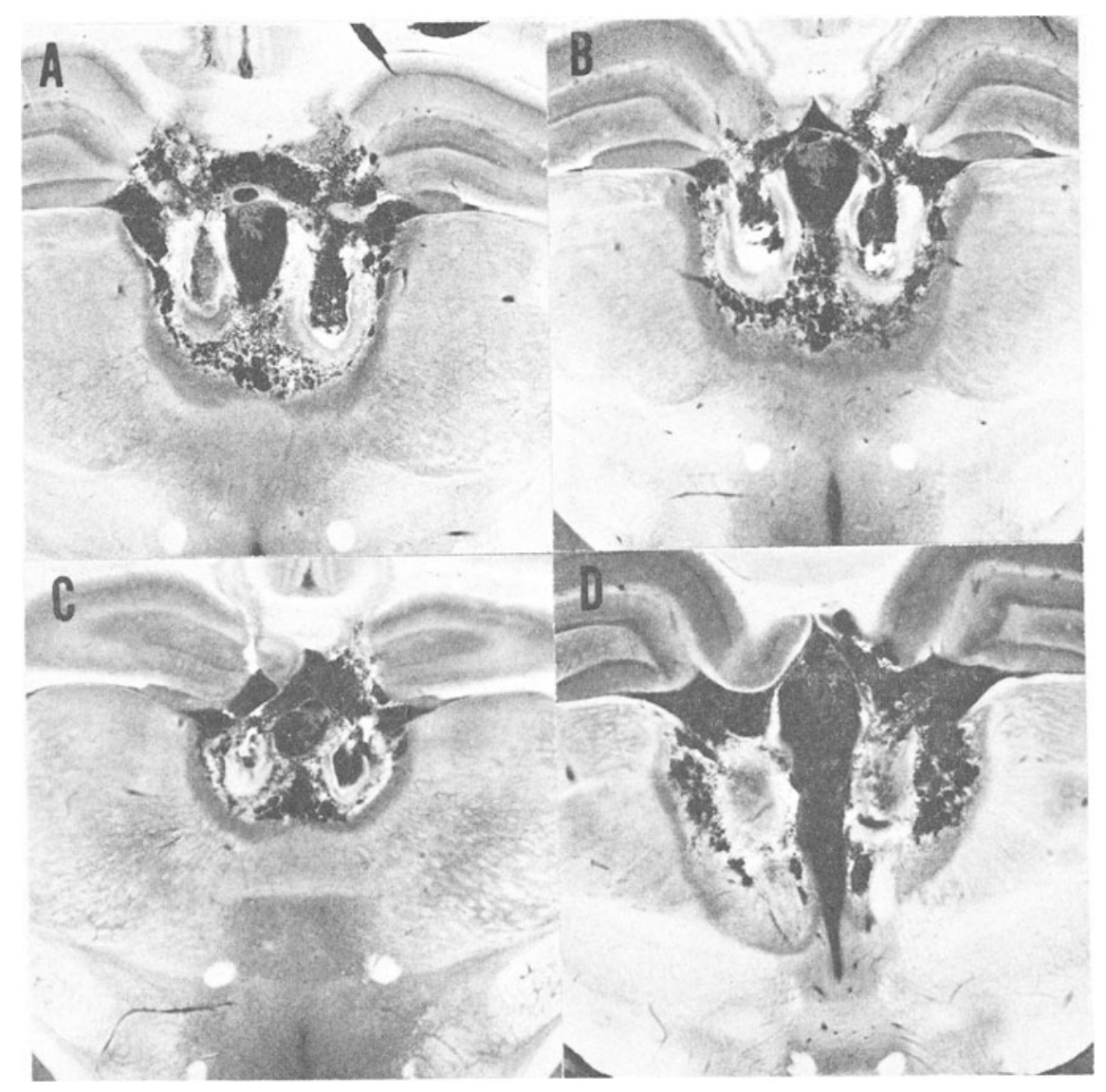

Figure 1. Photographs of unstained sections derived from four rats showing lesions to the habenular complex. Savings scores were as follows: (A) $0 \%$ and 100\%; (B) $67 \%$ and $89 \%$; (C) $100 \%$ and $100 \%$; (D) $100 \%$ and $100 \%$. 


\section{Thalamic Groups}

Dorsal sector. Of the six animals sustaining dorsomedial thalamic lesions, four earned savings scores below 50\% on Problem A (Figure 2). Retention scores earned on Problem B, however, were comparable to those earned by the controls.

Intermediate sector. There seems to be little doubt that lesions situated slightly more ventral than those received by Group DT tend to impair latchbox performance. Four of the six animals composing Group IT earned negative savings scores on both Problem A and Problem B (Figure 3).

Ventral sector. All four animals with thalamic lesions ventral to the massa intermedia exhibited moderate to severe retention losses on Problems A and B (Figure 4).

\section{Lesion Size vs. Lesion Locus}

No evidence of a "mass action" effect emerged from the data of this experiment; the rank-order correlations between the estimated size of the lesions and the extent of the retention deficits for all animals

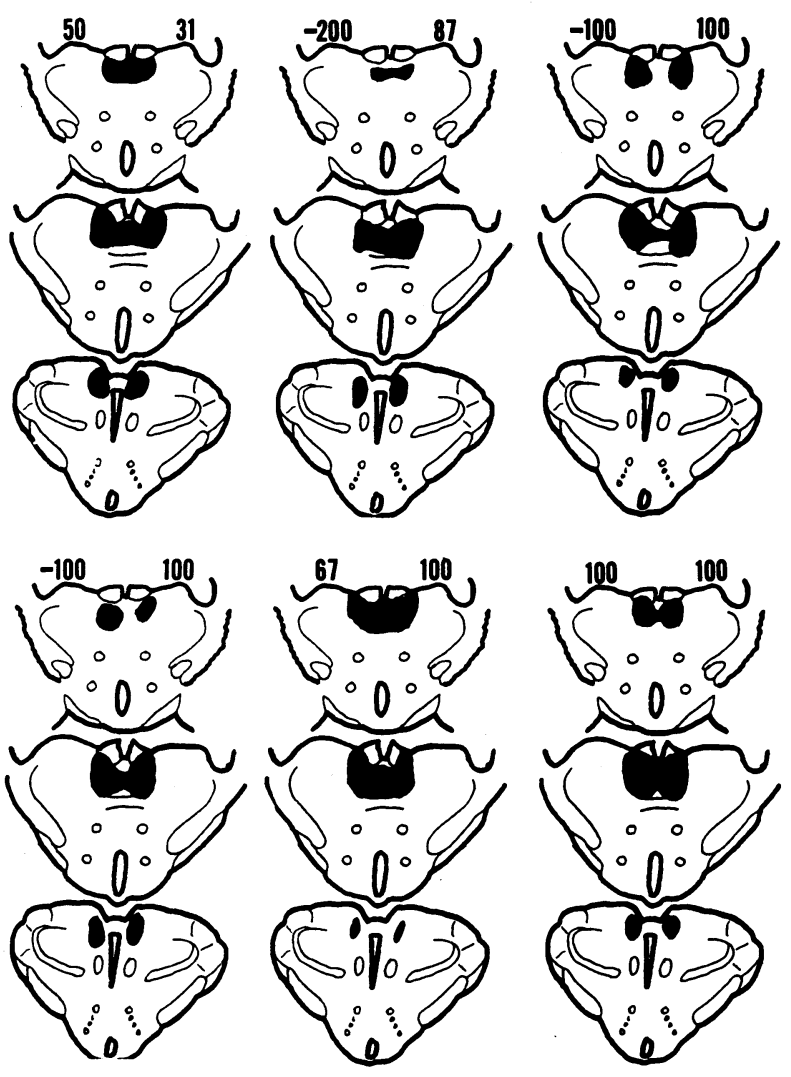

Figure 2. Frontal sections derived from the Thompson rat atlas showing lesions (blackened areas) in Group DT. Numbers to the left and right of each series of drawings indicate error savings scores earned on Problem A and Problem B, respectively.
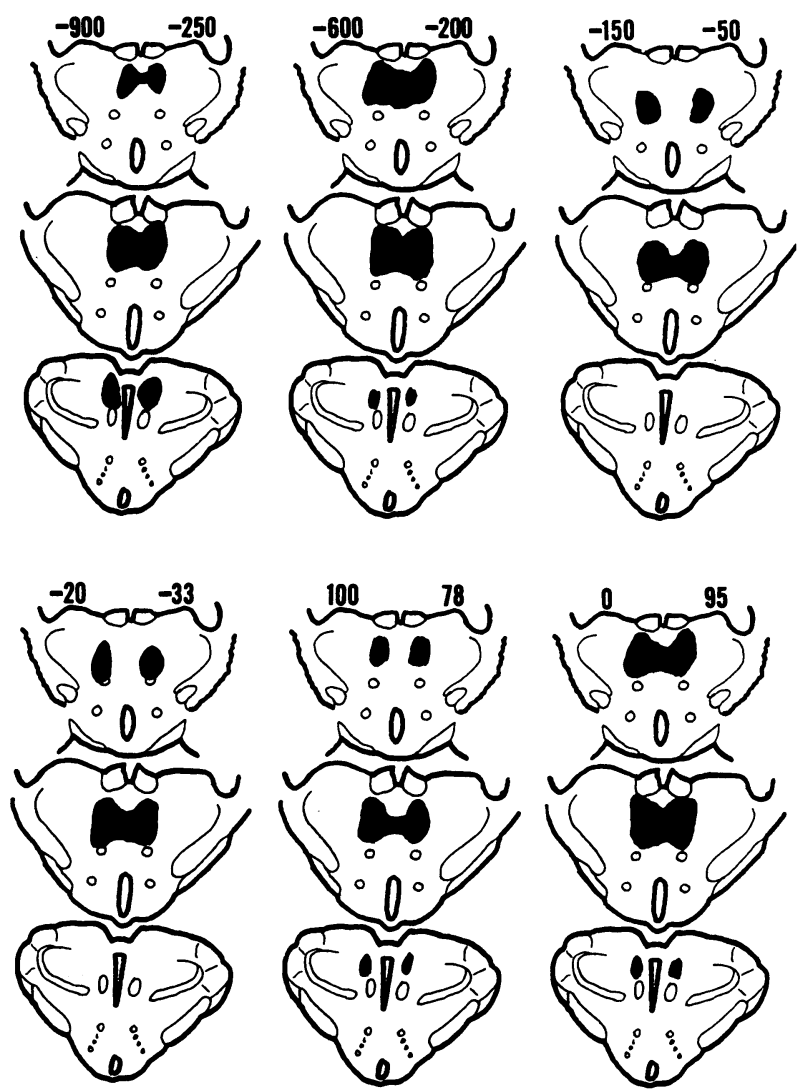

Figure 3. Frontal sections showing lesions in Group IT. See Figure 2 for description.

composing Groups DT, IT, and VT were -.08 for Problem A and - .02 for Problem B.

Separate rank-order correlations were computed between the estimated amount of damage to specific thalamic regions (mediodorsal, intralaminar, and ventromedial nuclei) and the degree of impairment in latchbox performance. As shown in Figure 5, the only significant correlation occurred between the amount of damage to the ventromedial nucleus and the extent of the retention loss on Problem B $(p=.01)$.

\section{Other Behavioral Observations}

\section{Motivational Disturbances}

Of the 20 brain-damaged subjects studied in this experiment, only two appeared to be timid and/or operating under low thirst motivation during the retention test on Problem A. One of these animals occasionally exhibited behavior indicative of low motivation (hesitancy in coming in contact with the latch or door and lack of vigor in manipulating the latch) during the course of the retention test on Problem B. Both of these animals belonged to 

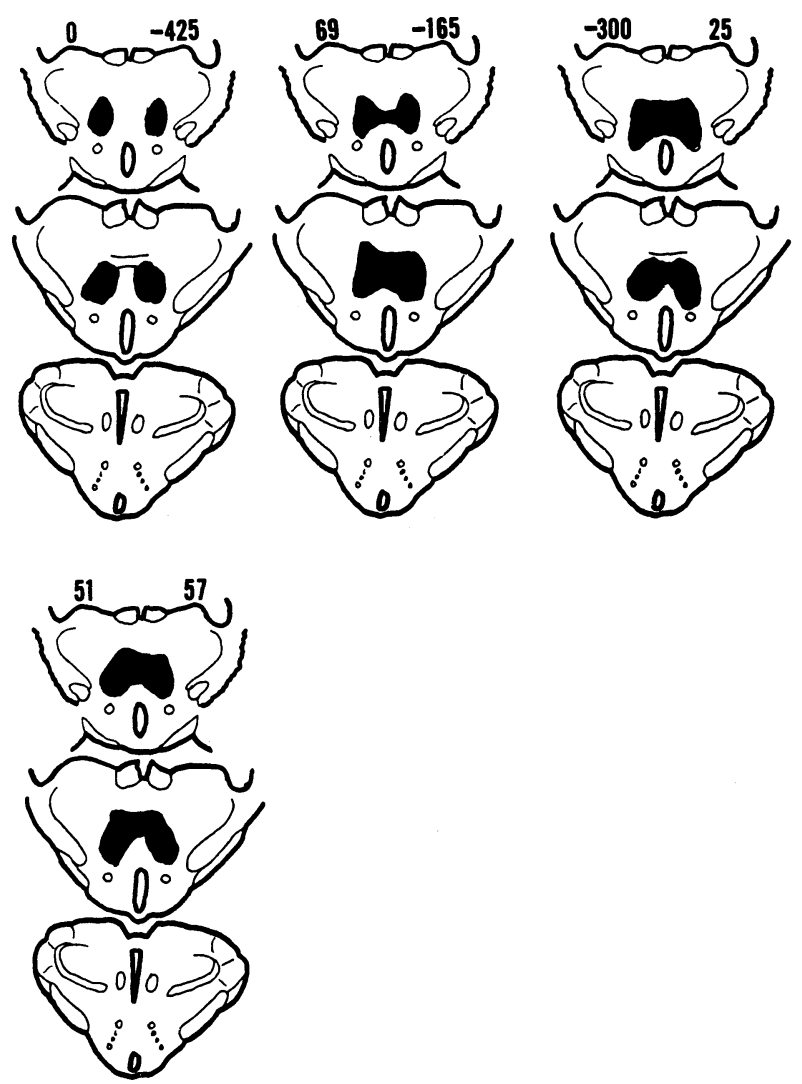

Figure 4. Frontal sections showing lesions in Group VT. See Figure 2 for description.

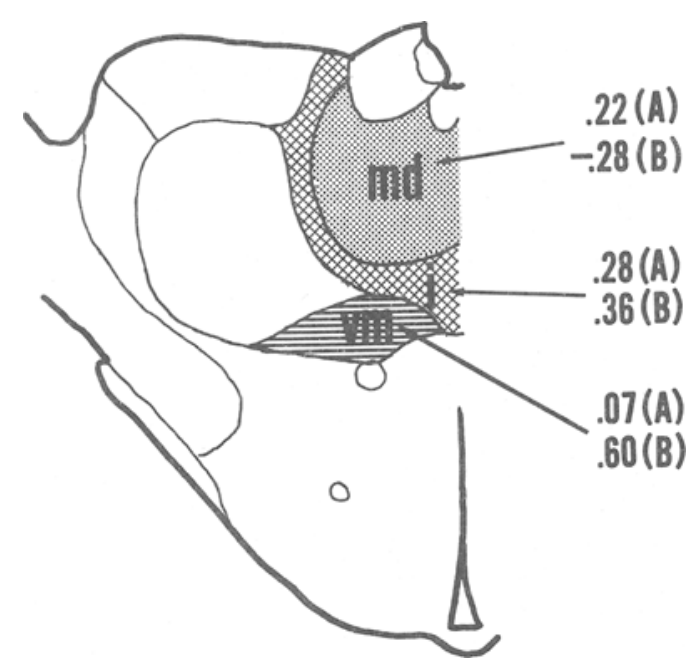

Figure 5. A frontal section taken from the Thompson rat atlas showing the three regions of the thalamus used to determine the relationship between the extent of bilateral damage and the retention scores earned on Problems A and B. Rank-order correlations are shown for the mediodorsal thalamic nucleus (md), intralaminar nuclei (i), and ventromedial thalamic nucleus (vm).
Group VT, and both earned negative savings scores on Problem B.

\section{Changes in Response Strategy}

As shown in a previous study (Thompson et al., 1979), those thalamic-damaged rats failing to employ the preoperatively used manipulative act during the retention test on a particular problem tended to exhibit defective retention of that problem (defective retention being defined as a savings score below the range established by the control group). This finding was confirmed in the present experimentof the six occurrences of a change in response strategy, defective retention scores were earned in all cases except one. However, it cannot be concluded that the use of the same manipulative act is associated with the achievement of normal savings scores (those falling within the range established by the control group). Of the 26 occurrences in which no change in response strategy was observed, only half were associated with normal savings scores.

\section{Quality of the Manipulative Response}

None of the brain-damaged (or control) rats of the present study showed any evidence of randomness or awkwardness of the manipulative act on those trials composing the criterion run on either problem. In most cases, the retention deficits seemed to have arisen from an inability to remember the appropriate response that operated a given latch. Six animals (three from Group IT, two from Group MT, and one from Group VT) that earned defective retention scores on Problem A and/or Problem B, however, did not fit into this category. These subjects appeared to remember how to manipulate the latch-they would unlock the door within $5 \mathrm{sec}-$ but rather than push the door open once the latch had been operated, they would persist for several seconds in pulling the latch toward themselves with their teeth. This specific disturbance in latchbox performance had not been detected in our earlier lesion studies on rats.

\section{DISCUSSION}

It is important to emphasize at the outset that defective retention scores earned on Problem B are most likely more indicative of injury to central motor mechanisms involved in the production of skilled movements than defective retention scores earned on Problem A. This is suggested by the following observations: (1) Rats with lesions of the motor cortex may achieve moderate to excellent savings scores on Problem A, but invariably earn negative savings scores on Problem B (Spiliotis \& Thompson, 1973; Thompson et al., 1979); (2) neodecorticate rats are able to relearn Problem A, but are unable to re- 
learn Problem B (Thompson et al., 1979); and (3) cerebellectomized rats, while failing to relearn either problem, make significantly more correct responses on Problem A than on Problem B (Thompson et al., 1979).

As shown in the present study, retention of Problem B was not significantly compromised by lesions to either the habenular complex or the mediodorsal thalamic nucleus. These findings would appear to be at variance with those of earlier studies (Thompson, 1979; Thompson et al., 1979), which reported retention deficits of discrete manipulative acts in rats subjected to habenular-dorsomedial thalamic lesions.

The existence of a critical focus within the ventral sector of the thalamus for latchbox deficits (see Figure 5) would appear to provide a satisfactory basis for explaining, at least in part, these discrepant results. For example, the so-called dorsomedial thalamic group studied in an earlier experiment (Thompson et al., 1979) was composed of rats with lesions that invaded thalamic tissue ventral to the massa intermedia. Similarly, the rat showing a severe card displacement deficit reported in another experiment (Thompson, 1979) suffered a habenular-medial thalamic lesion which extended beyond the boundaries of the intralaminar nuclei.

Obviously, the findings of the current study do not warrant the conclusion that the habenular complex and mediodorsal thalamic nucleus of the rat are totally devoid of motor function. Some evidence suggests that the habenular nuclei may be implicated in response inhibition (Waring \& Means, 1976) and that the mediodorsal nucleus may have a role in the initiation of voluntary movement (Vanderwolf, 1971). (It will also be recalled that Group DT exhibited a significant loss in retention of Problem A.) On the other hand, our data do suggest that neither the habenular complex nor the mediodorsal thalamic nucleus is part of the nervous substratum for the production of skilled (manipulative) movements in the rat.

Perhaps the most interesting finding of the present study concerns the identification of the medial thalamic focus for latchbox deficits. Based upon the rank-order correlations given in Figure 5, the ventromedial nucleus appears to be the critical medial thalamic site. This finding, however, must be interpreted with caution for two reasons. First, the intralaminar nuclei were not fully scanned with lesions in this experiment. Second, the deficits on Problem B arising from lesions to the ventromedial nucleus may be due, at least in part, to a motivational involvement. Nevertheless, it is becoming increasingly clear that the ventromedial nucleus may contribute in some significant way to the performance of manipulative acts in the rat. For example, displacing a barrier with the rhinarium in order to gain access to a goal object, a task that is sensitive to injuries to the sensorimotor cortex (Thompson, 1976), may be abolished in rats with ventromedial thalamic lesions as well (Thompson, 1978). Recent findings on the afferent and efferent connections of the ventromedial nucleus likewise suggest a role of this thalamic site in the expression of skilled movements. Thus, this nucleus is the recipient of fibers from several extrapyramidal structures, including the cerebellum (Faull \& Carman, 1978), substantia nigra (Faull \& Carman, 1968; Herkenham, 1979), and probably the globus pallidus (Carter \& Fibiger, 1978; Herkenham, 1979; Severin, Young, \& Massopust, 1976), and projects heavily to frontal cortical regions (Herkenham, 1979).

With regard to the functional significance of the ventromedial nucleus in latchbox performance, it is important to note that lesions of the ventromedial nucleus tend to produce deficits only on those tasks most likely to have a proprioceptive-kinesthetic basis, such as an incline plane discrimination (Thompson, Arabie, \& Sisk, 1976), a successive visual discrimination (Thompson, Hale, \& Bernard, 1980), and a maze habit (Thompson, 1974). Granting that learned manipulative acts also have a proprioceptive-kinesthetic basis (Konorski, 1967; Luria, 1966), then, it is reasonable to assume that the role of the ventromedial nucleus in latchbox performance is linked to the somatosensory regulation of skilled movements.

\section{REFERENCES}

Carter, D. A., \& Fibiger, H. C. The projections of the entopeduncular nucleus and globus pallidus in rat as demonstrated by autoradiography and horseradish peroxidase histochemistry. Journal of Comparative Neurology, 1978, 177, 113-124.

Faull, R. L., \& Carman, J. B. Ascending projections of the substantia nigra in the rat. Journal of Comparative Neurology, $1968,132,73-92$.

Faull, R. L., \& Carman, J. B. The cerebellofugal projections in the brachium conjunctivum of the rat. Journal of Comparative Neurology, 1978, 178, 495-518.

Herkenham, M. The afferent and efferent connections of the ventromedial thalamic nucleus in the rat. Journal of Comparative Neurology, 1979, 183, 487-518.

HerkenhaM, M., \& Nauta, W. J. H. Afferent connections of the habenular nuclei in the rat. A horseradish peroxidase study, with a note on the fiber-of-passage problem. Journal of Comparative Neurology, 1977, 173, 123-146.

Konorski, J. Integrative activity of the brain. Chicago: University of Chicago Press, 1967.

LURIA, A. R. Higher cortical functions in man. New York: Basic Books, 1966.

Severin, C. M., Young, P. A., \& Massopust, L. C. Pallidothalamic projections in the rat. Journal of Comparative Neurology, 1976, 166, 491-502.

Spiliotis, P. H., \& Thompson, R. The "manipulative response memory system" in the white rat. Physiological Psychology, $1973,1,101-114$.

Thompson, R. Localization of the "maze memory system" in the white rat. Physiological Psychology, 1974, 2, 1-17. 
Thompson, R. Card displacement response as affected by neocortical, cerebellar, and limbic forebrain lesions in the rat. Bulletin of the Psychonomic Society, 1976, 8, 101-102.

Tномpson, R. A behavioral atlas of the rat brain. New York: Oxford University Press, 1978.

Thompson, R. Dissociation of a visual discrimination task into incentive, location and response habits. Physiology \& Behavior, $1979,23,63-68$.

Thompson, R., Arabie, G. J., \& Sisk, G. B. Localization of the "incline plane discrimination memory system" in the white rat. Physiological Psychology, 1976, 4, 311-324.

Thompson, R., Gates, C. E., \& Gross, S. A. Thalamic regions critical for retention of skilled movements in the rat. Physiological Psychology, 1979, 7, 7-21.
Thompson, R., Hale, D. B., \& Bernard, B. A. Brain mechanisms concerned with left-right differentiation in the white rat. Physiological Psychology, 1980, 8, 309-319.

VANDERWOLF, C. H. Limbic-diencephalic mechanisms of voluntary movement. Psychological Review, 1971, 78, 83-113.

Waring, A. E., \& Means, L. W. Effect of medial thalamic lesions on emotionality, activity, and discrimination learning in the rat. Physiology \& Behavior, 1976, 17, 181-186.

(Received for publication February 23, 1981; accepted April 7, 1981.) 